Published in final edited form as:

Am J Geriatr Psychiatry. 2017 September ; 25(9): 931-938. doi:10.1016/j.jagp.2017.04.008.

\title{
Individualized Music Program is Associated with Improved Outcomes for U.S. Nursing Home Residents with Dementia
}

\author{
Kali Thomas, PhD, MA ${ }^{1,2,3}$, Rosa Baier, MPH ${ }^{1,3}$, Cyrus Kosar, MA ${ }^{1}$, Jessica Ogarek, MS ${ }^{1}$, \\ Alissa Trepman, MA, MPH ${ }^{3}$, and Vincent Mor, $\mathbf{P h D}^{1,2,3}$ \\ ${ }^{1}$ Center for Gerontology \& Healthcare Research, Brown University School of Public Health, \\ Providence, RI \\ ${ }^{2}$ Providence Veterans Affairs Medical Center, Providence, RI \\ ${ }^{3}$ Center for Long-Term Care Quality \& Innovation, Brown University School of Public Health, \\ Providence, RI
}

\begin{abstract}
Objectives-The objective of this study was to compare resident outcomes before and after implementation of an individualized music program, MUSIC \& MEMORYSM $(M \& M)$, designed to address the behavioral and psychological symptoms associated with dementia (BPSD).
\end{abstract}

Design: Setting—98 nursing homes (NHs) trained in the M\&M program during 2013 and 98 matched-pair comparisons.

Participants—Long-stay residents with Alzheimer's disease and related dementias (ADRD) residing in $M \& M$ participating facilities $(n=12,905)$ and comparison facilities $(n=12,811)$ during 2012-2013.

Intervention-M\&M is a facility-level quality improvement program that provides residents with music specific to their personal histories and preferences.

Measurements-Discontinuation of anxiolytic and antipsychotic medications, and reductions in behavioral problems and depressed mood in 2012 (pre-intervention) and 2013 (intervention), calculated using Minimum Data Set (MDS) assessments.

\footnotetext{
Corresponding Author: Kali Thomas, PhD, Brown University School of Public Health, Box G-S121-6, 121 S. Main Street, Providence, RI 029012, P: 401-863-9036, F: 401-863-3489, Kali_Thomas@Brown.edu. Note: The first two authors contributed equally.

Publisher's Disclaimer: This is a PDF file of an unedited manuscript that has been accepted for publication. As a service to our customers we are providing this early version of the manuscript. The manuscript will undergo copyediting, typesetting, and review of the resulting proof before it is published in its final citable form. Please note that during the production process errors may be discovered which could affect the content, and all legal disclaimers that apply to the journal pertain.

Meetings: AcademyHealth Annual Research Meeting, Boston, MA, June 2016

Conflicts of Interest: K. Thomas, PhD, MA; C. Kosar, MA; J. Ogarek, MS; A. Trepman, MA, MPH, and R. Baier, MPH have no potential conflicts of interest to declare.

Author Contributions: VM and RB obtained the data; KT and VM conceptualized and directed the analysis; CK completed the analysis; KT, RB, CK, and VM interpreted the results; KT and RB drafted the paper; CK, AT, and VM commented on drafts and approved the final paper.
} 
Results-The proportion of residents who discontinued antipsychotic medication use over a sixmonth period increased from $17.6 \%$ to $20.1 \%$ among M\&M facilities, while remaining stable among comparison facilities (15.9\% to $15.2 \%$ ). The same trend was observed for anxiolytic medications: discontinuation of anxiolytics increased in M\&M facilities (23.5\% to 24.4\%), while decreased among comparison facilities (24.8\% to 20.0\%). M\&M facilities also demonstrated increased rates of reduction in behavioral problems (50.9\% to 56.5\%) vs. comparison facilities (55.8\% to 55.9\%). No differences were observed for depressed mood.

Conclusions-These results offer the first evidence that the M\&M individualized music program is associated with reductions in antipsychotic medication use, anxiolytic medication use, and BPSD symptoms among long-stay NH residents with ADRD.

\section{Keywords}

Alzheimer's disease; antipsychotics; anxiolytics; dementia; long-term care; quality improvement

\section{INTRODUCTION}

\section{Objective}

Although national goals focus on preventing or curing Alzheimer's disease and related dementias (ADRD), ${ }^{1-3}$ disease prevalence is increasing with the aging population and is expected to reach 7.1 million people by $2025 .{ }^{4}$ Identifying and disseminating safe and effective interventions can therefore positively impact millions of people living with ADRD now and in the future. Evidence-based interventions are a particularly high priority for nursing homes $(\mathrm{NHs})$, where at least $50 \%$ of all residents have $\mathrm{ADRD}^{5}$ (about three times higher than among community-dwelling older adults ${ }^{6}$ ) and $80 \%$ of people with ADRD exhibit behavioral and psychological symptoms of dementia (BPSD) ${ }^{7}$ that can adversely affect their experiences and outcomes. ${ }^{8}$

Given the potential risks of using medications, such as antipsychotics, to address BPSD, ${ }^{9,10}$ finding effective non-pharmacological interventions is a high priority. Many patient-level non-pharmacological interventions exist, such as music therapy. Systematic reviews highlight the potential for music therapy to reduce agitation, ${ }^{11-14}$ aggression, ${ }^{12}$ anxiety, ${ }^{15}$ and behavioral symptoms, ${ }^{15,16}$ and improve mood. ${ }^{16}$ However, investigators note heterogeneous effects and generally weak study designs making the evidence, particularly for $\mathrm{NH}$ residents, somewhat inconclusive. ${ }^{17}$

One specific personalized music program, MUSIC \& MEMORYSM (M\&M), is growing in popularity. In M\&M, caregivers provide individuals diagnosed with ADRD music playlists that are tailored to their personal history of music preferences. ${ }^{18}$ The intervention's potential is illustrated in the award-winning 2014 documentary, Alive Inside, which shows residents with dementia moving, singing, and engaging with others when listening to their favorite music. ${ }^{19}$ However, its efficacy has not been rigorously established and little is known about its implementation and effectiveness in the nursing home setting. Therefore, the objective of this study was to examine the improvements that might be attributable to receiving M\&M among nursing home residents with ADRD. Building on the music therapy literature base and anecdotal evidence of the effectiveness of the M\&M program, we hypothesize that 
residents with ADRD in nursing homes that implemented M\&M would have increased rates of anti-psychotic and anxiolytic medication discontinuation, reductions in BPSD, and improvements in mood compared to residents in matched comparison facilities without the program.

\section{METHODS}

\section{Data}

Data for this study come from the Minimum Data Set (MDS) 3.0 and LTCfocUS.org for the years 2012 (pre-intervention) and 2013 (year of training, or intervention). The MDS is a federally-mandated assessment completed by clinicians at regular intervals (e.g., admission, discharge, quarterly, annually, and when a significant change occurs) for all $\mathrm{NH}$ residents. The resulting data include information about residents' diagnoses, treatments, symptoms, and medications. LTCfocUS.org is a Brown University database containing aggregated information from resident MDS assessments and facilities' Online Survey, Certification, And Reporting data, collected during the state inspections. ${ }^{21}$

\section{Sample}

The analytic sample included long-stay residents (i.e., identified as having at least one quarterly assessment, which suggested that they no longer were in the facility solely for post-acute, rehabilitative care) in facilities exposed to M\&M during calendar year 2013 and pair-matched comparison facilities. Because we were unable to determine which residents in the nursing home received the intervention, we identified our analytic sample as those who were likely to receive the intervention: residents with a diagnosis of ADRD who were not fully dependent in their Activities of Daily Living (an Activities of Daily Living (ADL) score less than or equal to 24; $)^{22}$ with some level of cognitive impairment (a Cognitive Function $\mathrm{Scale}^{23}$ greater than or equal to 2 ) and who were not receiving hospice in the facility or comatose. A diagnosis of ADRD came from the MDS Active Diagnoses Section (Section I). We included any individual who had an active diagnosis of Alzheimer's disease, non-Alzheimer's dementia, or one of the following ICD-9 codes listed: 290.0, 290.10, 290.11, 290.12, 290.13, 290.20, 290.21, 290.3, 290.40, 290.41, 290.42, 290.43, 294.0, 294.10, 294.11, 294.20, 294.21, 331.0, 331.11, 331.19, 331.2, 331.82, 331.7, or 797.

We obtained a list of facilities that were trained in M\&M during 2013 from MUSIC \& MEMORY, Inc. We used the 2012 data from LTCfocUS.org to match trained facilities to facilities with similar characteristics and resident composition. We excluded from our comparison all facilities trained by MUSIC \& MEMORY, Inc. in 2012 (pre-intervention) or 2014 ( $n=638$ ). Comparison facilities were matched exactly on geographical location (Medicare region), Medicare 5-Star Compare rating ${ }^{24}$ (category 2-3 and category 4-5, excluding facilities with the lowest rating, 1-Star), and for-profit status. Among these exact matched facilities, we calculated the absolute difference in facility occupancy rate, proportion of residents under the age of 65 years, proportion of residents with ADRD, total number of beds, and facility total staff hours/day/resident for M\&M and candidate matches. We averaged the absolute differences by facility and chose the candidate facility that on average had the smallest absolute difference, or the characteristics closest to those of the 
M\&M facility. Fewer than 10 homes were matched more than once and were reviewed by hand to choose the best match.

\section{MUSIC \& MEMORY Intervention}

$\mathrm{M} \& \mathrm{M}$ is a music program that builds upon evidence for individualized music synthesized in a 2013 review. ${ }^{20}$ In 2006, its creator, a social worker, decided to leverage inexpensive new technology (iPods) to create a personalized music program for people with ADRD. To implement M\&M, caregivers (e.g., healthcare providers or family members) create music playlists tailored specifically to each resident's personal history of music choices and preferences. $^{18}$

Before implementing M\&M, at least one staff member from each facility undergoes training by MUSIC \& MEMORY, Inc., a non-profit organization. Staff complete three live 90-minute webinars that focus on: 1) the benefits of personalized music and the legal boundaries for music sharing; 2) how to create personalized music playlists in iTunes ${ }^{\circledR}$; and 3) how to introduce the program and expand it incrementally over time. Afterwards, staff are encouraged to form teams charged with determining how best to implement M\&M, including selecting residents to receive iPods, pilot testing the process of researching music and providing it to residents, and expanding the intervention's use. Staff from certified facilities can access online and print resources for ideas and support about M\&M program implementation. These resources include a web site with best practices, one-on-one consultation, and coaching calls on different aspects of the program.

Cost for certification and training depends on the number of residents or the facilities in a corporation, with most paying \$250-\$1000 upfront and then \$200 per year. Some participants also receive "starter kits" with iPods and music gift cards, while others purchase all iPods or request donated equipment or money from families.

\section{Outcomes}

Antipsychotic and Anxiolytic Use-We examined whether or not a resident received any anxiolytic and/or antipsychotic medications during the seven days prior to an assessment as reported in Section N of the MDS. The MDS does not report specific drugs, only classes, including "antipsychotic" and "antianxiety" medications.

Behavioral Disturbance Presence and Frequency-We used the Aggressive Behavior Scale (ABS) to measure behavioral disturbance presence and frequency. ${ }^{25}$ The four items that compose the ABS come from the MDS Behavior section (Section E): physical, verbal, or other behavioral symptoms directed toward others, and rejection of care. Each item receives a score of $0-3$ indicating that the behavior was not exhibited in the last week (0) or that it occurred 1-3 days (1), 4-6 days (2), or daily (3). The MDS assessors make this assessment after reviewing the medical record, observing the resident, and consulting with staff and family members when possible. Total ABS scores range from 0-12 and are a resulting sum of the four items. The ABS is reported to have a Cronbach alpha between 0.79 and 0.93 , and is highly correlated with the Cohen-Mansfield Agitation Inventory (correlation coefficient $=0.72, \mathrm{P}<.001) .{ }^{25}$ 
Mood-We used the nine-item Patient Health Questionnaire (PHQ- $9^{\odot}$ ) severity score to measure mood. The PHQ-9 is a validated interview that screens for symptoms of depression and scores range from $0-27 .{ }^{26}$ For residents who were unable to complete the PHQ-9, we used scores from the PHQ-9 Staff Observation version (PHQ-9-OV ${ }^{\odot}$ ). Staff Assessment of Mood. The MDS assessor is instructed to interview the staff member who knows the resident best to complete the PHQ-9 OV.

\section{Data Analysis}

The exact timing of the M\&M implementation in 2013 was unknown. To circumvent this limitation, we compared changes in the use of medications, presence of behavioral symptoms, and mood within residents over a 180-day period in pre- and postimplementation years, assuming that $\mathrm{M} \& \mathrm{M}$ will have an effect over the 6-month period in 2013. In each year, the resident's 'baseline' assessment was the first in which study inclusion criteria were met. Outcomes measured at baseline were subtracted from their measurements at the assessment six months later (or closest to this point). Thus, for each resident we identified whether antipsychotics and anxiolytics were discontinued at 180-days, and whether symptoms of behavior disturbances and depressed mood were reduced (indicated by a positive difference between 180-day PHQ-9 and ABS scores) in pre- and post- implementation years in facilities with and without M\&M. We then used conditional logistic regression to identify whether rates of medication withdrawal, behavioral symptom reduction, and improvement in mood were different between the pre-intervention and intervention periods among M\&M sites vs. pair-matched comparison facilities. We modeled each individual outcome as a function of treatment status, year, the interaction of year and treatment, and facility fixed effects. The coefficient of the interaction term represents the differences-in-differences estimator, and when significant indicates that outcomes in the preintervention and intervention period were different for $\mathrm{M} \& \mathrm{M}$ sites and comparison facilities. The conditional logistic model was chosen to account for the matched-pair design, and by including facility fixed effects the effect of $M \& M$ was identified on the variation in outcomes across individuals residing in the same facility. Analyses were conducted using Stata MP version 14.1 (StataCorp, College Station, TX). Null hypotheses were tested with a two-tailed alpha of 0.05 .

Brown's Center for Long-Term Care Quality \& Innovation (Q\&I Center), which tests interventions to improve post-acute and long-term care, has access to MDS data under a CMS data use agreement. The Brown Institutional Review Board approved our use of these data to evaluate quality improvement projects.

\section{RESULTS}

During the pre-intervention year, matched comparison facilities had a lower proportion of non-whites and lower average lengths of stay of long-stay residents with ADRD (Table 1). Comparison facilities were also more likely to have long-stay residents with ADRD who had a higher mood score, lower rates of behavioral disturbances, and higher rates of anxiolytic medication use. 
Difference-in-differences estimates showed statistically significant improvements for several measures among residents residing in facilities that participated in M\&M (Table 2).

Specifically, we found that the difference in the rates of discontinued antipsychotic and anxiolytic medication use and reduced behavioral problems was greater in $\mathrm{M} \& \mathrm{M}$ facilities compared to pair-matched comparison facilities. Among M\&M facilities, the proportion of residents who discontinued antipsychotic medication increased from $17.6 \%$ in 2012 to $20.1 \%$ in 2013 , while remaining stable among comparison facilities (15.9\% to $15.2 \%$,). The proportion of residents who discontinued anxiolytic medication increased slightly among M\&M facilities (23.5\% to $24.4 \%$ ), while decreased among comparison facilities (24.8\% to $20.0 \%$ ). Residents in M\&M facilities also demonstrated increased rates of improvement in behavioral symptoms (50.9\% exhibiting behavior improvement in 2012 to $56.5 \%$ exhibiting improvements in 2013) compared to residents in comparison facilities (55.8\% in 2012 and $55.9 \%$ in 2013). In 2012, the mean ABS score for all residents in M\&M facilities was 0.84 $(\mathrm{SD}=0.83)$ and $0.67(\mathrm{SD}=1.52)$ in comparison facilities (see Table 1). In the following year, the mean $\mathrm{ABS}$ score was $0.74(\mathrm{SD}=1.72)$ in $\mathrm{M} \& \mathrm{M}$ facilities and $0.63(\mathrm{SD}=1.44)$ in comparison facilities. There was no statistically significant difference in the differences between M\&M and comparison facilities observed for changes in mood.

\section{DISCUSSION}

Results from this study offer the first evidence that the M\&M individualized music program may be associated with reductions in antipsychotic and anxiolytic medication use as well as improvement in BPSD among NH residents with ADRD. While we did not observe relative improvements in mood, this retrospective study builds upon the literature base supporting the potential for personalized music therapy to reduce antipsychotic and anxiolytic use and improve BPSD, ${ }^{11-16}$ particularly among NH residents. ${ }^{27-29}$

M\&M offers a low-cost, non-pharmacological solution to the growing burden that the projected increase in ADRD will place on NH providers. The program is popular and spreading: by the end of 2015, thousands of provider sites across the world had become certified. Twelve states currently have M\&M NH demonstration projects underway, including four state-sponsored projects (California, Ohio, Texas, and Wisconsin). Its popularity stems, in part, from the fact that it offers a non-pharmacological alternative to anti-psychotic medications, which can pose significant risks and are the focus of a national patient safety campaign. It is also low-cost: start-up costs for implementing a M\&M program include staff training and iPod and music purchases. Apart from those costs, ongoing implementation requires only staff time to identify residents that would benefit from music, research those individuals' music preferences, and incorporate the use of the iPods into daily care routines.

Our work adds to the literature by establishing the effect of a personalized music program in reducing BPSD, anxiolytic, and antipsychotic medication use in a population of long-stay NH residents with ADRD. Previously, meta-analyses found that music-related interventions were associated with reductions in depression, ${ }^{15}$ anxiety ${ }^{30}$ and BPSD ${ }^{15,30}$ among individuals with dementia. However, these findings were not specific to the $\mathrm{NH}$ population. While our findings were not consistent with previous studies that have shown that music 
effectively improves depression in older adults with ADRD, we did find reductions in behavioral disturbance presence and frequency, as well as reduction in the use of anxiolytic and antipsychotic medications.

This study has many strengths, including a larger sample size than any previously-reported outcomes of individualized music therapy, a matched comparison group, and validated, routinely-collected outcome measures. However, it is a retrospective analysis constrained by the fact that we do not know when facilities were trained during 2013, when or how they implemented the program, and which residents received iPods. We may underestimate the effect by examining outcomes for all long-stay residents with ADRD, when only a subset actually received the intervention. Future work to evaluate this program will consider random assignment of facilities to participate in the M\&M program or to serve as comparisons and should include an implementation evaluation that allows us to characterize the residents selected by staff to participate, the delivery of the intervention, and any immediate effects that may be recognizable through residents' participation in the M\&M intervention.

As with any program, illuminating the "black box" of implementation is integral to understanding its effectiveness and generalizability. MUSIC \& MEMORY, Inc. reports that the programs' use varies widely across NHs, including which residents are selected to receive an iPod, where iPods are stored, and how often residents have access to them. Future research is needed to characterize factors associated with effective implementation in order to standardize staff training and program implementation to optimize program performance and ensure optimal resident outcomes.

We note several additional limitations. First, we assume that the program was in place for all of 2013 and that all long-stay residents with dementia received iPods. Residents may have participated in the intervention before or after their baseline MDS assessments in that year, or may not have participated at all. However, these assumptions result in estimates that are attenuated rather than biased upward. Second, while we attempted to match M\&M with comparison facilities based on meaningful observable characteristics, we were unable to fully control for differences that may have affected the outcomes of interest. For example, facilities that chose to take on a new intervention, in this case M\&M, may also be interested in adopting other innovative or best practices for dementia care, such as increasingly adopting non-pharmacological strategies and minimizing the use of antipsychotics or anxiolytics. In addition to differences we found in some measured facility characteristics (e.g., resident race and length of stay), it is possible that $M \& M$ facilities are inherently different from those that chose not to participate in ways that we are unable to measure with our data.

\section{CONCLUSION}

In summary, this study offers the first evidence that the M\&M program may be associated with reductions in the use of antipsychotic and anxiolytic medications, as well as BPSD symptoms among NH residents with ADRD. While more insight is required to understand which residents are most likely to benefit from this particular music therapy program and 
what improvement they experience, our findings signal that in the aggregate, the $M \& M$ program is associated with improvement in the experience of care provided to residents with ADRD in NHs.

\title{
Acknowledgments
}

\begin{abstract}
Vincent, $\mathrm{PhD}$ is a founder and on the board of directors of PointRight, Inc. an information services company serving nursing homes on quality measurement and improvement. Vincent, $\mathrm{PhD}$ is also a stock holder for PointRight, Inc. and Navi Health. Vincent, $\mathrm{PhD}$ receives no research funding, data or consultation on his research from PointRight or NaviHealth. Vincent, PhD also received honoraria from the Alliance for Health Care Quality.

Sponsor's Role: Not applicable - none

This analysis was conducted by Brown University’s Center for Long-Term Care Quality \& Innovation, which partners with providers and other innovators to rigorously test and disseminate interventions to improve post-acute and long-term care. Learn more at brown.edu/go/innovation. Dan Cohen, MSW of MUSIC \& MEMORY, Inc. provided a list of M\&M certified facilities and Michael Brondino, $\mathrm{PhD}$, of the University of Wisconsin shared findings from the Wisconsin state demonstration project's analysis.
\end{abstract}

Source of Funding: National Institutes of Health [grant number P01AG027296].

\section{References}

1. Alzheimer's Association. The National Alzheimer's Project Act (NAPA). 2010. http://napa.alz.org/ national-alzheimers-project-act-backgroun. Accessed April 14, 2016

2. Office of the Assistant Secretary for Planning and Evaluation (ASPE). National Plan to Address Alzheimer's Disease: 2015 Update. 2015. https://aspe.hhs.gov/national-plan-address-alzheimer $\%$ E2\%80\%99s-disease-2015-update. Accessed April 14, 2016

3. U.S. Department of Health and Human Services. 2014-2015 Alzheimer's Disease Progress Report: Advancing Research Toward a Cure. 2015. https://www.nia.nih.gov/alzheimers/publication/ 2014-2015-alzheimers-disease-progress-report/introduction. Accessed April 14, 2016

4. Alzheimer's Association. 2015 Alzheimer's Disease Facts and Figures: Includes a Special Report on Disclosing a Diagnosis of Alzheimer's Disease. 2015. https://www.alz.org/facts/downloads/ facts_figures_2015.pdf. Accessed April 14, 2016

5. CDC. , editor. U.S. Department of Health and Human Services Centers for Disease Control and Prevention, National Center for Health Statistics. Long-Term Care Services in the United States: 2013 Overview. Vol. 3. Washington, DC: 2013.

6. Plassman BL, Langa KM, Fisher GG, et al. Prevalence of dementia in the United States: the aging, demographics, and memory study. Neuroepidemiology. 2007; 29(1-2):125-132. [PubMed: 17975326]

7. Margallo-Lana M, Swann A, O'Brien J, et al. Prevalence and pharmacological management of behavioural and psychological symptoms amongst dementia sufferers living in care environments. Int J Geriatr Psychiatry. 2001; 16(1):39-44. [PubMed: 11180484]

8. Kales HC, Gitlin LN, Lyketsos CG. Assessment and management of behavioral and psychological symptoms of dementia. BMJ. 2015; 350:h369. [PubMed: 25731881]

9. Mittal V, Kurup L, Williamson D, et al. Risk of cerebrovascular adverse events and death in elderly patients with dementia when treated with antipsychotic medications: a literature review of evidence. Am J Alzheimers Dis Other Demen. 2011; 26(1):10-28. [PubMed: 21282274]

10. U.S. Department of Health \& Human Services. Safety: Atypical Antipsychotic Drugs. 2005. http:// www.fda.gov/Safety/MedWatch/SafetyInformation/SafetyAlertsforHumanMedicalProducts/ ucm150688.htm. Accessed April 14, 2016

11. Livingston G, Kelly L, Lewis-Holmes E, et al. A systematic review of the clinical effectiveness and cost-effectiveness of sensory, psychological and behavioural interventions for managing agitation in older adults with dementia. Health Technol Assess. 2014; 18(39):1-226. v-vi. 
12. Whear R, Abbott R, Thompson-Coon J, et al. Effectiveness of mealtime interventions on behavior symptoms of people with dementia living in care homes: a systematic review. J Am Med Dir Assoc. 2014; 15(3):185-193. [PubMed: 24405641]

13. Craig J. Music therapy to reduce agitation in dementia. Nurs Times. 2014; 110(32-33):12-15.

14. Livingston G, Kelly L, Lewis-Holmes E, et al. Non-pharmacological interventions for agitation in dementia: systematic review of randomised controlled trials. Br J Psychiatry. 2014; 205(6):436442. [PubMed: 25452601]

15. Ueda T, Suzukamo Y, Sato M, et al. Effects of music therapy on behavioral and psychological symptoms of dementia: a systematic review and meta-analysis. Ageing Res Rev. 2013; 12(2):628641. [PubMed: 23511664]

16. McDermott O, Crellin N, Ridder HM, et al. Music therapy in dementia: a narrative synthesis systematic review. Int J Geriatr Psychiatry. 2013; 28(8):781-794. [PubMed: 23080214]

17. Brasure, M., Jutkowitz, E., Fuchs, E., et al. Nonpharmacologic Interventions for Agitation and Aggression in Dementia. Rockville, MD: 2016.

18. Memory M. Music \& Memory. https://musicandmemory.org/. Accessed April 14, 2016

19. Rossato-Bennett, M. Alive Inside. 2014. http://www.aliveinside.us/. Accessed April 14, 2016

20. Gerdner LA, Buckwalter KC. Musical Memories: translating evidence-based gerontological nursing into a children's picture book. J Gerontol Nurs. 2013; 39(1):32-41.

21. Shaping Long-Term Care in America Project. http://ltcfocus.org/. Accessed Feb 4, 2016

22. Morris JN, Fries BE, Morris SA. Scaling ADLs within the MDS. J Gerontol A Biol Sci Med Sci. 1999; 54(11):M546-553. [PubMed: 10619316]

23. Thomas KS, Dosa D, Wysocki A, et al. The Minimum Data Set 3.0 Cognitive Function Scale. Med Care. 2015 Epub ahead of print.

24. Centers for Medicare \& Medicaid Services. Design for Nursing Home Compare Five-Star Quality Rating System Technical Users' Guide. Feb. 2015 https://www.cms.gov/Medicare/ProviderEnrollment-and-Certification/CertificationandComplianc/downloads/usersguide.pdf. Accessed April 14, 2016

25. Perlman CM, Hirdes JP. The aggressive behavior scale: a new scale to measure aggression based on the minimum data set. J Am Geriatr Soc. 2008; 56(12):2298-2303. [PubMed: 19093929]

26. Saliba D, DiFilippo S, Edelen MO, et al. Testing the PHQ-9 interview and Observational Versions (PHQ-9 OV) for MDS 3.0. J Am Med Dir Assoc. 2012; 13(7):618-625. [PubMed: 22796361]

27. Ray KD, Mittelman MS. Music therapy: A nonpharmacological approach to the care of agitation and depressive symptoms for nursing home residents with dementia. Dementia (London). 2015

28. Werner J, Wosch T, Gold C. Effectiveness of group music therapy versus recreational group singing for depressive symptoms of elderly nursing home residents: pragmatic trial. Aging Ment Health. 2015:1-9.

29. Raglio A, Bellelli G, Traficante D, et al. Efficacy of music therapy in the treatment of behavioral and psychiatric symptoms of dementia. Alzheimer Dis Assoc Disord. 2008; 22(2):158-162. [PubMed: 18525288]

30. Chang YS, Chu H, Yang CY, et al. The efficacy of music therapy for people with dementia: A meta-analysis of randomised controlled trials. J Clin Nurs. 2015; 24(23-24):3425-3440. [PubMed: 26299594] 


\section{Table 1}

Facility Characteristics for M\&M Sites and Pair-Matched Comparison Facilities During the Pre-Intervention Year, 2012

\begin{tabular}{|c|c|c|c|}
\hline Characteristic & $\begin{array}{l}\text { M\&M Sites } \\
(\mathbf{N}=\mathbf{9 8})\end{array}$ & $\begin{array}{l}\text { Pair-Matched Comparison Facilities } \\
\qquad(\mathbf{N}=98)\end{array}$ & Test, df, p-value \\
\hline Total beds, mean (sd) & $175.6(132.1)$ & $164.4(116.2)$ & $\mathrm{t}=-0.63, \mathrm{df}=194, \mathrm{p}=0.53$ \\
\hline Occupancy, mean \% (sd) & $88.3(11.4)$ & $89.0(9.2)$ & $\mathrm{t}=-0.51, \mathrm{df}=194, \mathrm{p}=0.61$ \\
\hline Not-for-profit, n (\%) & $64(65.3)$ & $64(65.3)$ & $X^{2}=0.00, \mathrm{df}=1, \mathrm{p}=1.00$ \\
\hline Medicare, mean \% (sd) & $14.9(11.2)$ & $13.3(9.0)$ & $\mathrm{t}=-1.09, \mathrm{df}=14, \mathrm{p}=0.28$ \\
\hline$<65$ years, mean \% $(\mathrm{sd})$ & $13.7(14.9)$ & $12.1(13.2)$ & $\mathrm{t}=-0.83, \mathrm{df}=194, \mathrm{p}=0.41$ \\
\hline Member of Chain, $\mathrm{n}(\%)$ & $34(34.7)$ & $39(39.8)$ & $X^{2}=0.55, \mathrm{df}=1, \mathrm{p}=0.46$ \\
\hline Staffing, mean hours/residents/day (sd) & $3.7(0.7)$ & $3.7(0.7)$ & $\mathrm{t}=0.20, \mathrm{df}=194, \mathrm{p}=0.84$ \\
\hline 5 Star Nursing Home Compare Rating & & & $X^{2}=0.00, \mathrm{df}=1, \mathrm{p}=1.00$ \\
\hline 2 or $3, \mathrm{n}(\%)$ & $48(49.0)$ & $48(49.0)$ & \\
\hline 4 or $5, \mathrm{n}(\%)$ & $50(51.0)$ & $50(51.0)$ & \\
\hline Overall Star-Rating mean (sd) & $3.5(1.2)$ & $3.5(1.1)$ & $\mathrm{t}=0.44, \mathrm{df}=194, \mathrm{p}=0.66$ \\
\hline Residents Included in Analytic Sample & $n=6298$ & $n=6278$ & \\
\hline Age, mean years (sd) & $84.4(9.7)$ & $84.6(9.2)$ & $\mathrm{t}=1.52, \mathrm{df}=12574, \mathrm{p}=0.13$ \\
\hline Female Sex, n (\%) & $4627(73.5)$ & $4554(72.5)$ & $X^{2}=1.38, \mathrm{df}=1, \mathrm{p}=0.24$ \\
\hline Non-White Race, n (\%) & $1978(31.4)$ & $1225(19.5)$ & $\mathrm{t}=2.32, \mathrm{df}=12574, \mathrm{p}<0.001$ \\
\hline \multicolumn{4}{|l|}{ Length of Stay in Nursing Home, mean } \\
\hline days (sd) & $635.8(791.1)$ & $581.3(773.4)$ & $\mathrm{t}=-3.91, \mathrm{df}=12574, \mathrm{p}<0.001$ \\
\hline \multicolumn{4}{|l|}{ Medication Use, Behaviors, and Mood } \\
\hline Any Anxiolytic use, n (\%) & $886(14.1)$ & $1038(16.5)$ & $X^{2}=14.75, \mathrm{df}=1, \mathrm{p}<0.001$ \\
\hline Any Antipsychotic use, n (\%) & $1831(29.1)$ & $1876(29.9)$ & $X^{2}=0.97, \mathrm{df}=1, \mathrm{p}=0.33$ \\
\hline Any Behavioral Problem, n (\%) & $1679(26.7)$ & $1604(25.6)$ & $X^{2}=1.98, \mathrm{df}=1, \mathrm{p}=0.16$ \\
\hline \multicolumn{4}{|l|}{ Behavioral Disturbance Presence and } \\
\hline Frequency ${ }^{\dagger}$, mean (sd) & $0.8(1.8)$ & $0.7(1.5)$ & $\mathrm{t}=-5.33, \mathrm{df}=12574, \mathrm{p}<0.001$ \\
\hline $\operatorname{Mood}^{*}$, mean (sd) & $2.9(3.7)$ & $3.1(4.1)$ & $\mathrm{t}=3.96, \mathrm{df}=11934, \mathrm{p}<0.001$ \\
\hline \multicolumn{4}{|c|}{ Measured using the Patient Health Questionnaire 9-Item Depression Screener or PHQ-9-OV (range: 0-27; higher=worse); } \\
\hline easured using the Aggressive Behav & ale (range: 0 & ; higher=worse) & \\
\hline
\end{tabular}

Am J Geriatr Psychiatry. Author manuscript; available in PMC 2018 September 01. 


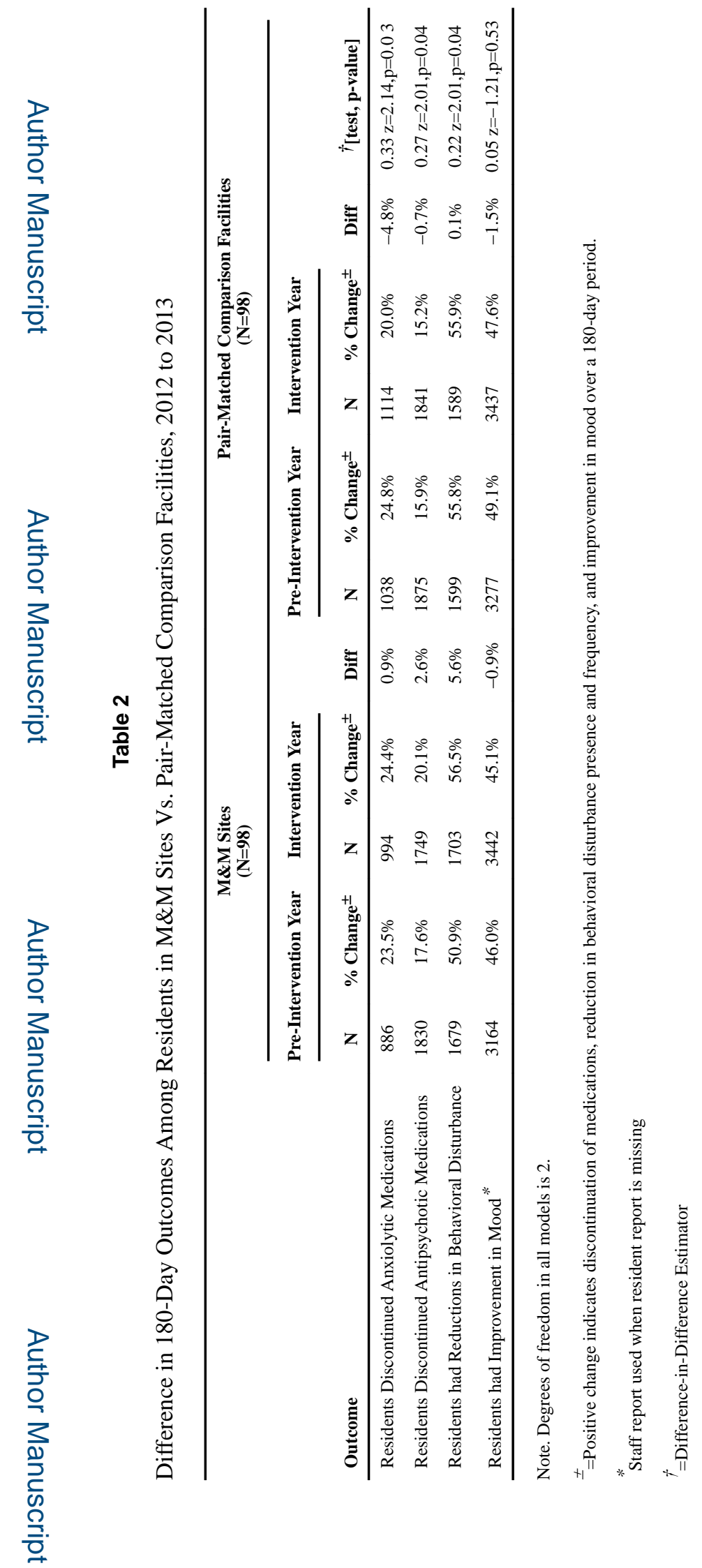

Am J Geriatr Psychiatry. Author manuscript; available in PMC 2018 September 01. 